\title{
A Clínica, a Epidemiologia e a Epidemiologia Clínica
}

Neste ensaio, pretendo desenvolver a tese de que o modo como a epidemiologia constrói o seu objeto de conhecimento, que equivale ao seu modo de produção de saber, é inadequado, se usado sem mediações, para constituir o discurso da clínica. Isto implica um enfrentamento da proposta da chamada "epidemiologia clínica" - talvez a mais importante ideologia científica na área da saúde desde a "medicina preventiva" -, na tentativa de organizar uma argumentação que critique a lógica do projeto clínicoepidemiológico.

Os manuais da "epidemiologia clínica"1,2,3 apresentam as seguintes dimensões como características dessa proposta de disciplina integrativa: a) uma certa teoria do diagnóstico clínico, baseada na avaliação da validade e na confiabilidade dos procedimentos de identificação de caso; b) uma metodologia para a construção do conhecimento etiológico a partir de estratégias observacionais de pesquisa em pequenos grupos; c) uma metodologia correspondente para estudos de eficácia e efetividade de procedimentos terapêuticos; d) a proposição de uma "clinimetria", estru-

\footnotetext{
* Ph.D., professor adjunto do Departamento de Medicina Preventiva da FAMED-UFBA. Professor visitante do Departamento de Antropologia da Universidade da Califórnia em Berkeley, EUA. Pesquisador 1-A do Conselho Nacional de Desenvolvimento Científico e Tecnológico - CNPq.

1. Fletcher P.H., Fletcher S.W., Wagner E., Clinical Epidemiology - The Essentials. Baltimore, Williams \& Wilkins, 1982.

2. Sackett D., Haynes B., Tugwell P.,Clinical Epidemiology. Boston, Little, Brown \& Co., 1985.

3. Jénicek M., Cléroux R., Épidémiologie Clinique. Québec, Edisen Inc., 1985.
} 
turada com o emprego de modelos probabilísticos de tomada de decisões, para o estabelecimento do prognóstico clínico.

Com exceção do esboço de uma certa propedêutica de base quantitativa, denominada clinimetria ${ }^{4}$ aparentemente nada há de novidade em termos de aplicação da metodologia epidemiológica corrente a problemas clínicos. Apesar da evidente falta de originalidade, trata-se de uma proposta admiravelmente orquestrada. Em termos práticos, grupos de epidemiologia clínica têm se organizado em competição com os núcleos de pesquisa epidemiológica, antagonizando-os em praticamente todos os níveis da práxis institucional. Tal "luta ideológica" tem se desenrolado em todas as frentes, desde a formação de recursos humanos (disputando a primazia pelo ensino de conteúdos epidemiológicos nas escolas médicas) até a própria produção de conhecimento (competindo ferozmente por financiamentos de pesquisa), desde o controle das sociedades científicas até o monopólio dos veículos de divulgação.

Em termos conceituais, tal competição expressa-se em uma luta aberta pelo arbítrio da cientificidade. Os grupos da Universidade de Yale e da Mcmaster, pioneiros do movimento da epidemiologia clínica, ${ }^{5,6,7}$ têm sustentado, em diversas oportunidades, que o "paradigma experimental" 8 deve ser tomado como padrão exclusivo de rigor científico para a pesquisa em saúde.

É evidente que tais movimentos articulam-se estreitamente com uma tendência de tecnificação da prática médica, que vem cada vez mais reduzindo-se à aplicação de tecnologias para o reconhecimento e manuseio de quadros patológicos. Nessa direção, a prática clínica tem se pautado quase exclusivamente por um enfoque individualizado e biologicista, com pouca ênfase no desenvolvimento conceitual da medicina e de suas ciências básicas. Trata-se de um projeto a-histórico e acrítico, que se defronta com impasses e dilemas próprios, como espero adiante demonstrar.

Inicialmente, pretendo discutir de uma maneira sistematizada os múltiplos vínculos, empíricos e teóricos, entre a clínica ${ }^{9}$ e a epidemiologia,

4. Idem.

5. Feinstéin A., "Why clinical Epidemiology?" Clinical Research 20:821-5, 1972.

6. Feinstein A., "An additional basic science for clinical medicine, I-Iv." Annals of Internal Medicine 99:393-397, 554-560, 705-712, 843-8848, 1983.

7. Sackett D., Haynes B., Tugwell P., op. cit.

8. Horwitz R.I., "The experimental paradigm and observation studies of cause-effect relationships in clinical medicine". J. Chron Dis 40:91-9, 1987.

9. Apenas para simplificar, chamaremos genericamente de clínica à clínica médica. Recordemos que há outro tipo de clínica, a psicanalítica, por exemplo, cujo referencial é bastante diverso, senāo oposto, ao da clínica médica. 
juntamente com as suas oposições conceituais e metodológicas. Em seguida, buscarei avaliar o grau de legitimidade epistemológica da proposta de hibridizaçāo característica da "epidemiologia clínica", particularmente em relação às suas tentativas de tecnificar o processo de tomada de decisão na prática diagnóstica e terapêutica da clínica.

\section{Subordinada porém complementar}

Preliminarmente, é importante acentuar o caráter subsidiário da epidemiologia em relação ao saber clínico. Segundo Gonçalves ${ }^{10}$ a complementaridade entre estes dois campos do conhecimento encontra-se "garantida pela univocidade do conceito de doença, que representa no nível do saber a integração das práticas clínicas e de saúde pública". A fonte da heterogeneidade fundamental que permite a construção do objeto de conhecimento na epidemiologia encontra-se na diferenciação potencial entre pessoas doentes e sadias, o que é possibilitado pela abordagem clínica de indivíduos membros da população.

A clínica e a epidemiologia encontram-se vinculadas epistemologicamente. Ambas tratam de corpos sociais: enquanto a clínica trata do sujeito considerado em suas particularidades, o caso, o um, a epidemiologia aborda o coletivo, busca a generalidade, o grupo de casos, o todos. A atuação individualizada da prática clínica não deixa de ser uma intervenção sobre corpos sociais, ${ }^{11}$ através de "encontros singulares", na medida em que trata de homens concretos, em contextos sócio-históricos. A epidemiologia, mesmo no seu enfoque mais tradicional que reforça o biologicismo da clínica ao reduzir o social ao mero conjunto de indivíduos, ${ }^{12}$ também trata de corpos social e historicamente definidos, nesse caso corpos sociais coletivos.

A mais marcante expressão de um laço tão forte será talvez evidente na fonte do determinante do objeto da clínica, localizável no campo epidemiológico, e na definição do objeto da epidemiologia, subordinado ao campo da clínica. Como sabemos, esta subordinação se revela desde a

10. Gonçalves R.B., "Reflexão sobre a articulaçāo entre a investigação epidem iológica e a prática médica: A propósito das doenças crônicas degenerativas. In: Costa D. (org.), Epidemiologia: Teoria e Objeto. São Paulo, Hucitec-Abrasco, 1990, p. 39-85.

11. Apesar disso, alguns campos disciplinares subsidiários à clínica médica fornecem um exemplo contundente da dicotomizaçáo corpo físico/corpo social. A anatomia patológica e a fisiologia, por exemplo, examinam e sistematizam o conhecimento sobre este "corpo" como se ele fosse um objeto natural, ou seja, não atravessado pela linguagem e pela cultura, e portanto a-social.

12. Gonçalves R.B., op. cit. 
constituição do próprio objeto epidemiológico (doentes em populações), dado que a identificação dos doentes é produzida, em última instância, pela abordagem clínica.

Tanto a clínica médica quanto a epidemiologia necessitam de teorias produzidas fora de si mesmas para se afirmarem como disciplinas científicas. A clínica, para lidar com sujeitos humanos, busca esses conhecimentos no campo da patologia. A epidemiologia, no tratamento de grupos humanos, tem utilizado, com sucesso relativo, o saber produzido pelas ciências sociais a fim de subsidiar teorias da sociedade para compreender processos coletivos de saúde-doença. ${ }^{13}$ Nesse aspecto, não se encontra realmente uma teoria clínica da doença, tanto quanto não faz nenhum sentido qualquer teoria epidemiológica da sociedade.

Metodologicamente, clínica e epidemiologia interagem. Em primeiro lugar, servem-se mutuamente como fontes de problemas científicos, de modelos explicativos e de hipóteses de pesquisa. Em segundo lugar, os instrumentos de investigação epidemiológica são construídos, quase sempre, a partir de padrões oriundos da observação clínica. Ao mesmo tempo, a validade e a confiabilidade dos procedimentos diagnósticos da clínica têm sido testados por meio da metodologia epidemiológica. Nesse aspecto específico, será sempre instrutivo rever a origem da nosologia e os fundamentos dos exames ditos complementares, no começo da clínica. ${ }^{14}$

Desenhos de pesquisa originalmente desenvolvidos para a investigação clínica vêm sendo aperfeiçoados, cada vez mais, pela epidemiologia, no momento em que são ampliados e aplicados em populações. Tais avanços são quase prontamente devolvidos à clínica, que os tem absorvido com sucesso no processo de configurar uma "metodologia de investigação clínica". A competente história da evolução da pesquisa epidemiológica realizada por Susser ${ }^{15}$ é exemplar desse processo de fertilização mútua, especialmente em relação aos estudos longitudinais. O estudo de caso-controle ${ }^{16}$ parece mais ser um exemplo da tendência oposta, uma estratégia de pesquisa concebida no campo epidemiológico que logo tornou-se hegemônica na investigação clínica etiológica.

Até aqui, mencionei somente alguns pontos de intersecção dos dois campos disciplinares. Gostaria agora de discutir algumas das suas

13. Susser M., Epidemiology, Health \& Society —Selected Papers. Nova York, Oxford Univ. Press, 1987.

14. Almeida Filho N., Epidemiologia sem números -Uma introdução crítica a ciência epidemiológica. Rio, Campus, 1989.

15. Susser M., op. cit.

16. Cole P., "The Evolving Case-Control Study". J. Chron. Dis. 32:15-27, 1979. 
contradições potenciais, inicialmente abordando oposições de caráter epistemológico.

\section{Oposições epistemológicas}

O que é essencial no raciocínio clínico ao lidar com um problema de saúde-doença? Ou, mais concretamente, qual a atitude objetiva do clínico frente a uma pessoa que o procura com um conjunto de sinais e sintomas?

Sempre que o quadro de sinais/sintomas lhe dá elementos suficientes, ele (ou ela) firma um diagnóstico. Quando o perfil sintomatológico não se mostra suficientemente claro, como ocorre na maioria das vezes, o clínico levanta hipóteses diagnósticas, partindo para a realização de exames ditos complementares. Esses testes têm a finalidade de produzir novos dados que, integrados às outras informações clínicas, serão enquadrados em uma "entidade mórbida" estabelecida ao longo do processo da observação clínica. ${ }^{17,18}$ Nessa fabricação do conhecimento sobre um caso clínico, muitas vezes o diagnosticador tem que agir de um modo que pode ser considerado "intuitivo". Geralmente, o seu roteiro de trabalho consiste, porém, em estudar os casos particulares empregando formulações gerais previamente definidas (que é a nosografia estabelecida), tratadas como se fossem leis universais. O modo de raciocínio do clínico é, portanto, fundamentalmente dedutivo..$^{19,20,21}$

O raciocínio epidemiológico parte da observação de casos ocorridos em uma dada população, agrupa-os segundo séries de variáveis (individuais, temporais, geográficas, socioeconômicas, culturais), analisa o que eles têm em comum, estabelece associações (potencialmente, fatores de risco), buscando explicações tipo causalidade para a ocorrência da patologia. Dessa forma, a partir da observação de casos particulares de um determinado evento de saúde-doença, os epidemiologistas derivam inferências sobre o que terá ocorrido em outras amostras, grupos ou populações e que eventos futuros provavelmente ocorrerão naquelas populações, caso as características e condições observadas sejam mantidas. Portanto, o conhecimento epidemiológico é produzido através de um método predominantemente indutivo. ${ }^{22,23}$

17. Foucault M., O nascimento da clínica. São Paulo, Forense, 1979.

18. Gonçalves R.B., op. cit.

19. Murphy E., The Logic of Medicine. Baltimore, Johns Hopkins Univ. Press, 1965.

20. Black D.A., The Logic of Medicine. Edinburgh, Oliver \& Boyd, 1968.

21. Ledermann E.K., Philosophy and Medicine. Cambridge, Gower, 1986.

22. Susser M., op. cit. 
É claro que a clínica também usa freqüentemente o raciocínio indutivo, especialmente durante as etapas precoces de criação de novas figuras nosológicas, em que se articulam as informações propedêuticas geradas por um conjunto de eventos particulares. ${ }^{24}$ Por seu turno, a epidemiologia reserva um lugar para o raciocínio dedutivo, particularmente nos estágios iniciais de construção das questões de pesquisa. ${ }^{25}$ Tem existido um forte movimento na direção de uma epidemiologia não-indutiva, apelidado de “epidemiologia popperiana", ${ }^{26,27}$ porém o principal da pesquisa epidemiológica e suas aplicações parece indelevelmente marcado pela indução. ${ }^{28}$.

Assumindo tais posturas metodológicas, a clínica se integra melhor a um referencial de determinação causal e/ou estrutural. Nessa perspectiva, o seu modo privilegiado de raciocínio se constrói a partir da doença, ou do sintoma, em direção à suposta causa. $\mathrm{O}$ foco do olhar clínico é sempre dirigido para o âmbito individual, sendo o caso idealmente considerado em sua singularidade, ou seja, no que tem de particular e exclusivo. Afinal, a representação básica será a noção de subconjunto de sujeitos doentes será a representação básica, elemento matematicamente definido nos termos estabelecidos por Miettinen. ${ }^{29}$

Em relação ao esquema de construção do objeto, a clínica parte do seu determinante típico (que se origina no campo da epidemiologia), efeito de "fatores de risco" sobre o sujeito singular, visualizando um processo de produção individual da doença. Só a partir daí constrói-se um objeto clínico, resultante do reconhecimento de um agente mórbido (ou conjunto de agentes) atuando sobre o corpo individual, mesmo que socialmente determinado, provocando um certo quadro de modificações, exacerbações, processos novos e até lesões em vários níveis. Trata-se aqui do objeto doença, construído em um processo de raciocínio modal enquadrado nos parâmetros da fisiopatologia, tendo a biologia como sua legitimadora em termos de cientificidade. ${ }^{30}$

Já do ponto de vista da epidemiologia, o esquema equivalente de construção do objeto de conhecimento inicia-se pelo determinante epide-

23. Pearce N. e Crawford-Brown D., "Critical Discussion in Epidemiology: Problems with the Popperian Approach". Journal of Clinical Epidemiology 42:177-85, 1989.

24. Hunter K.M., "A Science of Individuals: Medicine and Casuistry". The Journal of Medicine and Philosophy 14:193-212, 1989.

25. Pearce N. e Crawford-Brown D., op. cit.

26. Buck C., "Popper's Philosophy for Epidemiologists". Int. J. Epidem. 4:159-168, 1975.

27. Weed D., "On the logic of causal inference". American Journal of Epidemiology 123(6):965-79, 1986.

28. Susser M., op. cit.

29. Miettinen O., Theoretical Epidemiology. Nova York, John Wiley \& Sons, 1985.

30. Canguilhem G., O normal e o patológico. Forense-Universitária. Rio de Janeiro, 1982. 
miológico (ou complexo de determinantes: a configuração de risco) que incide sobre uma dada população, produzindo um subconjunto de doentes, especificados como tal a partir da perspectiva clínica. O entendimento desse processo tem sido convencionalmente constituído em torno de modelos explicativos de base clínica, não obstante o enorme esforço que as abordagens chamadas socioepidemiológias têm feito para desenvolver modelos teóricos próprios. De todo modo, pelo menos como pano de fundo, a legitimação científica da ciência epidemiológica tem sido buscada nas ciências matemáticas.

Entretanto, os pontos de contradição entre estes dois campos de conhecimento não se esgotam no nível da conceitualização. As oposições encontradas na esfera dos processos concretos de produção de conhecimento, com uma natureza mais restritamente metodológica portanto, podem ser ainda mais marcantes, como veremos a seguir.

\section{Oposições metodológicas}

Na sua prática concreta, a clínica tem um compromisso predominante com a intervenção sobre a saúde individual e, como um corolário, com o desenvolvimento de tecnologias efetivas para o apoio às estratégias diagnósticas e terapêuticas. No nível da pesquisa, em última instância, a clínica busca produzir diagnósticos e prognósticos que sejam tratados como evidência científica. Em contrapartida, o compromisso fundamental da epidemiologia é com a produção de conhecimento em si, um tipo de conhecimento que, apesar das limitações do seu próprio método, busca, no fim de contas, elucidar a determinação do processo saúde-doença em geral. ${ }^{31}$ Para isso, a epidemiologia se interessa explicitamente pela descrição dos padrões de distribuição da ocorrência em massa de doenças em populações. Fornece, ainda, subsídios para o desenvolvimento e o aperfeiçoamento de medidas preventivas e práticas de saúde coletiva.

Com isso não quero dizer que os clínicos pouco se interessam pelas correlações sociais e históricas dos processos coletivos da saúde-doença, ou que os epidemiologistas nada têm a ver com os processos biológicos individuais. Na verdade, gostaria aqui de propor a existência de éticas opostas nas práticas respectivas. Na clínica, dado o seu compromisso ético primordial com a saúde de cada paciente, ${ }^{32}$ a incerteza não deve ser

31. Miettinem O., op. cit.

32. Cassel E.J., "The conflict between the desire to know and the need to care for the patient". In: Spicker S (ed.), Organism, Medicine, and Metaphysics. Boston, D. Reidel Publ. Co., 1978, p. 57-72. 
pbstáculo para a ação. "Na dúvida, faça alguma coisa" — diz um antigo aforisma clínico. Na epidemiologia, considerando o seu compromisso ético com a busca de um conhecimento positivo inalcançável, a dúvida incidental inviabiliza proposições afirmativas. Em outras palavras, a produção do conhecimento científico não tolera a incerteza; portanto, "na dúvida, não se pode afirmar".

O caráter idealista de ambas as éticas, científica e médica, que de modo equivalente as aliena do mundo real da prática, não muda a substância deste argumento, que indica essências em contradição, apontadas para direções radicalmente opostas. Nem o grande esforço de construção de proposições usando um jargão probabilístico, observado em ambos os campos, atenua o antagonismo aqui exposto. Enunciados probabilísticos de fato propiciam matéria adicional para o processo decisório de avaliação da prova científica, seja para o estabelecimento de diagnóstico, prognóstico ou tratamento, seja para a derivação de inferências ou predições. Entretanto, o critério para a validade da evidência científica não é primariamente a significância estatística, mas um conjunto bastante complexo e compreensivo de operações heurísticas, particulares a cada disciplina científica. No que se refere à avaliação da abordagem clínica, em vez da significância estatística, deve-se procurar estabelecer primeiro a significância clínica de uma dada evidência. ${ }^{33}$

A mirada da clínica sobre o seu objeto, olhar que transforma fenômenos indiferenciados em objeto específico de conhecimento (ou intervenção), tem para cada caso um caráter particular e subjetivo, restrito, na maioria das vezes, aos seus próprios limites de visão. Num movimento de dedução-intuição, o profissional clínico busca apreender a "essência patológica" de cada caso em estudo, utilizando métodos diagnósticos complexos e exaustivos. ${ }^{34}$ As estratégias modais da pesquisa clínica se caracterizam pelo enfoque particularizado, acentuando o que há de singular no sujeito investigado, com uma aproximação mais bem caracterizada como intensiva-profunda. A clínica tem como pretensão última saber tudo sobre o um (enquanto projeto idealizado, evidentemente).

Dada a especificidade de seu objeto - doentes em população - a epidemiologia não pode pretender abordagens "personalizadas" de cada caso. A disciplina é empurrada na direção de critérios universais pela sua própria vocação histórica e epistemológica. Seus desenhos de pesquisa tendem ao geral e à "extensidade", na medida em que procuram a extra-

33. Sackett D., Haynes B., Tugwell P., op. cit.

34. Black D.A., op. cit. 
polação para o todo. Os desenhos observacionais característicos da epidemiologia não se encontram submetidos a um controle rígido, como ocorre com os desenhos experimentais, tomados como paradigma da pesquisa clínica. ${ }^{35}$ A justificativa básica desta "flexibilidade" é simplesmente que a referência da investigação epidemiológica encontra-se em populações reais, ${ }^{36}$ coletivos concretos não replicáveis em contextos laboratoriais controlados. Para fins de diagnóstico em massa, necessários à identificação de doentes em populações, a epidemiologia tem desenvolvido ou adaptado instrumentos de aceitável especificidade e sensibilidade, capazes de produzir dados consistentes e confiáveis é que, ao mesmo tempo, tenham boa aplicabilidade. Para atender a tais requisitos, os instrumentos da pesquisa epidemiológica costumam ser simplificados e padronizados, permitindo sua utilização em larga escala, controlando ou mensurando a extensão do erro nos resultados obtidos.

A clínica utiliza, como fonte de informação, pequenos grupos de casos ou mesmo casos isolados. Tais grupos de casos, homogeneamente constituídos (como um ideal de precisão diagnóstica, evidentemente), formarão a chamada "casuística", base da "experiência clínica" ${ }^{37} \mathrm{O}$ tamanho desses grupos variará de acordo com a frequiência de ocorrência ou de identificação do fenômeno mórbido estudado. Em investigações clínicas, o tamanho dos grupos em estudo poderá depender também da conveniência do pesquisador, tendo em vista a viabilização do estudo em termos operacionais, especialmente recursos humanos, e materiais, e tempo de acompanhamento.

Tipicamente, as coletas de dados clínicos seguem um padrão recorrente e repetido, sendo necessário, muitas vezes, a observação diária de cada caso, produzindo um enorme volume de dados por caso. Dessa forma, pelo menos em perspectiva, busca-se em geral uma apreensão totalizadora do processo de doença em desenvolvimento. $O$ dado clínico tem como referência fundamental a sua homogeneidade frente aos quadros sindrômicos estabelecidos, assumindo portanto o estatuto de sinal ou sintoma. Para que seja considerada como dado clínico (atingindo a "significância clínica"), uma informação deverá se mostrar suficientemente sensível para o reconhecimento da doença. Ou seja, precisa idealmente apresentar-se de uma forma homogênea em todos os casos — na prática, basta estar presente em uma maioria razoável de doentes.

35. Horwitz R.I., op. cit.

36. Miettinen O., "The clinical trial as a paradigm for epidemiologic research".J. ClinEpidemiol 42:491-6, 1989.

37. Hunter K.M., op. cit. 
Por outro lado, a epidemiologia trabalha com populações ou amostras, condenada à ambição da "lei dos grandes números". ${ }^{38}$ Por esta pretensão, a produção de dados epidemiológicos normalmente realiza-se em poucas oportunidades de trabalho de campo, exigindo instrumentos capazes de fornecer apenas os dados essenciais para a análise (que, por sua vez, já será pré-dirigida para estabelecer associações previamente admitidas como hipóteses). Nesse processo, a referência fundamental para o dado epidemiológico é sua representatividade perante o conjunto amostral da população.

Nos elementos de análise também residem oposições entre os campos disciplinares considerados. A investigação epidemiológica parte de hipóteses previamente levantadas (mesmo que implícitas) e refutáveis. O efeito das variáveis em estudo pode ser controlado no próprio desenho da pesquisa, pela restrição do âmbito da observação, ou na fase de análise de dados, através do emprego de técnicas de ajuste. A principal (mas não a única) fonte de certeza da investigação epidemiológica é a significância estatística, ${ }^{39}$ também expressa quantitativamente por meio do famigerado índice chamado de "valor p". Tem havido muita discussão sobre o sentido preciso da testagem da significância estatística. ${ }^{40,41}$ Porém, para os propósitos da presente argumentação, basta admitir que se trata simplesmente de uma medida da confiança do investigador no potencial de inferência dos resultados do estudo, pelo menos da amostra para a população de referência.

O raciocínio clínico, por sua vez, parte de hipóteses sucessivas e plausíveis que potencialmente levam a proposições diagnósticas e/ou prognósticas. ${ }^{42} \mathrm{O}$ controle da investigação é dado a priori pelo próprio desenho de estudo (como no caso dos ensaios clínicos), ou pela estratégia das aproximações sucessivas, presente no do processo terapêutico. As fontes de certeza da clínica são a consistência e a coerência. Um certo resultado de pesquisa terá validade clínica quando puder ser inscrito em uma dada casuística, compondo a homogeneidade de um conjunto estabelecido de casos similares. Dessa forma, contribuirá para o refinamento de uma "experiência clínica" consistente pela ampliação quantitativa e qualitativa da casuística. Além disso, um resultado clinicamente relevante é

38. Almeida Filho N., op. cit.

39. Rothman K., Modern Epidemiology. Boston, Little Brown, 1986.

40. Howson C., Urbach P., Scientific Reasoning. The Bayesian Approach. La Salle, Open Court Publ., 1989.

41. Oakes M., Statistical Inference. Chestnut Hill, Epidemiology Resources Inc., 1990.

42. Murphy E., op. cit. 
aquele que se revela coerente com objetos de doença preexistentes, ou seja, que faz sentido dentro dos modelos ontológicos de doença que estão na base de cada abordagem clínica em particular. ${ }^{43}$

Em síntese, não obstante as complexas relações dialéticas entre esses campos de conhecimento, como vimos acima, as suas formas privilegiadas de aproximação aos objetos da saúde-doença não podem ser reduzidas uma à outra. A tese subjacente a este ensaio é que débito não significa submissão. A forma como a epidemiologia constrói seu objeto de conhecimento, que equivale ao seu particular modo de produção de conhecimento, não pode ser subsumido pelo discurso clínico. Nem vice-versa: a epidemiologia não é "a clínica das populações", tanto quanto a clínica nunca se tornará a "epidemiologia dos indivíduos". A negação desta impossibilidade original é que parece fundamentar a proposição de uma "epidemiologia clínica". ${ }^{44}$

\section{As críticas da epidemiologia clínica}

Alvan Feinstein, talvez o mais competente formulador teórico da epidemiologia clínica, em uma interessante provocação publicada em 1988 na prestigiada revista $S$ cience, propõe que a metodologia observacional característica da investigação epidemiológica não é capaz de produzir o conhecimento etiológico rigoroso, necessário nos termos desse suposto paradigma para a produção científica em saúde. Feinstein ${ }^{45}$ argumenta ainda que, se o critério último de atribuição de causalidade a evidências epidemiológicas consiste na confirmação experimental, não se justifica a realização de estudos de desenho observacional prospectivo ou retrospectivo, em geral dispendiosos e altamente propensos a resultados inconclusivos. Este e outros autores ${ }^{46,47}$ privilegiam os desenhos de pesquisa controlados para a construção do conhecimento clínico-epidemiológico, em detrimento de estudos de coorte e estudos de caso-controle. Implícita em tal proposta encontra-se a posição de que a epidemiologia seria dispensá-

43. Gonçalves R.B., "Contribuição à Discussāo sobre as Relaçōes entre Teoria, Objeto e Método em Epidemiologia”. Anais, I Congresso Brasileiro de Epidemiologia, Campinas, 1990, p. 346-361.

44. Em termos retóricos, trata-se de um escandaloso oxímoro, conforme denunciado por Last (1988) [Last J., Editorial. Journal of Public Health Policy (summer):1988.

45. Feinstein A., "Scientific Standards in Epidemiologic Studies of the Menace of Daily Life". Science 242:1257-63, 1988.

46. Sackett D., Haynes B., Tugweel P., op. cit.

47. Horwitz R.I., op. cit. 
vel, e mesmo contra-indicada, para a produção de conhecimento científico em geral sobre o fenômeno doença. ${ }^{48}$

O que mais incomoda nesta provocaçāo é que aí se expõe, com toda a crueza, a pretensão dos epidemiologistas de produzir conhecimento causal em um campo cuja práxis tem construído historicamente um objeto distinto dos objetos da clínica e das ciências básicas da saúde. É como se Horwitz e Feinstein jogassem na cara dos epidemiologistas a seguinte questão: "Se vocês admitem que só se pode estabelecer a natureza causal de uma dada associação depois da demonstração experimental, por que realizar estudos seccionais, de caso-controle e de coorte, custosos e com resultados controversos, e não passar diretamente aos ensaios clínicos controlados?" Ou, mais grosseiramente: "Para quê epidemiologia?"

Reações a tal bateria de críticas ${ }^{49}$ têm se detido em aspectos secundários da questão, como equívocos e incorreçōes nos exemplos apontados pelo enfant terrible Feinstein, sem enfrentar a argumentação em seus aspectos substanciais, sem notar a estreiteza da concepção de ciência aí revelada, sem sequer assinalar o uso equivocado da categoria kuhniana de paradigma pelos "epi-clínicos". Apenas Miettinen ${ }^{50}$ sugere, ainda que timidamente e sem maior elaboração, que esse conjunto de críticas situa-se no âmbito restrito de um dado paradigma.

Pelo lado metodológico, esquecem, tanto críticos como defensores, que a arquitetura dos próprios ensaios clínicos não apresenta diferenças formais em relação aos estudos longitudinais. Trata-se meramente de estudos com uma expectativa de resultados invertida, em que coortes (homogeneizadas pela característica da doença em questão) são expostas não a fatores de exposição "naturais", e sim a intervençōes artificialmente provocadas, verdadeiros fatores de risco de manifestações em geral opostas à doença, como cura ou melhora. Reciprocamente, os estudos de coorte nada mais são do que desenhos experimentais reconstruídos a posteriori, respeitando as restriçōes impostas pelas situações reais de pesquisa, com um reduzido controle do investigador sobre os fatores em ação. Pelo lado conceitual, quanto mais controlada a situação experimental, maior será o teor de "artificialidade" da investigação, reduzindo potencialmente a validade externa dos seus resultados que, supōe-se, deverão ser aplicáveis ao

48. Feinstein A., op. cit.

49. Savitz D., Greenland S., Stolley P., Kelsey J., "Scientific Standards of Criticism: A Reaction to Standards in Epidemiologic Studies of the Menace of Daily Life, by A.R. Feinstein". Epidemiology $1 ; 78-83,1990$

50. Miettinen O., Editorial — "Precision and Insight". Journal of Clinical Epidemiology 43(3):211-214, 1990. 
mundo real, a sujeitos históricos, sadios e doentes, e não referidos ao microcosmo asséptico e controlado dos laboratórios. Este é um argumento antigo - levantado na década de 1930 por Theobald $\mathrm{Smith}^{51}$ para defender a pesquisa médica fora dos ambientes clínicos - porém extremamente atual, merecendo um lugar de destaque neste debate.

\section{Clinimetria: novidade antiga?}

Aparentemente, a proposta da clinimetria seria então a única dimensão da epidemiologia clínica que não se configura como uma simples aplicação de técnicas de pesquisa epidemiológica a questōes clínicas. A proposta consiste basicamente na adoção de um raciocínio probabilístico, com base em modelos estocásticos, para a tomada de decisōes sobre o comportamento de doenças em indivíduos. Em outras palavras, trata-se da substituição dos modelos causais, mecanicistas ou estruturais, característicos do pensamento clínico, por modelos de determinaçāo probabilística, que até entāo pareciam típicos do raciocínio epidemiológico. Nāo é nenhuma novidade a passagem direta, ou reduçāo, de um modelo causal puro para uma estrutura de explicação derivada da aplicação de expectativas aleatórias a eventos observados. Muito pelo contrário, significa talvez a mais primária (e primeira) tentativa de sistematizar o pensamento clínico em bases inferenciais, fazendo da medicina a primeira ciência humana aplicada. ${ }^{52,53,54}$

A indução já havia sido proposta como método privilegiado do raciocínio sistemático da ciência desde o século XVI, com Bacon e Galileu. Posteriormente, defrontou-se com inúmeras dificuldades para a sua aplicação concreta no âmbito da chamada "história natural",s5 face à relativa escassez de regularidades unívocas e absolutas encontradas na observação dos seres vivos. Porém, no momento em que a prática científica passou a adotar as regras de indução, a filosofia já antecipava críticas cruciais à fundamentação lógica desse método, propondo a existência de um "problema da induçāo". ${ }^{56,57}$

51. Smith T., Parasitism and Disease. Princeton, Princeton Press, 1934.

52. Foucault M., op. cit.

53. Clavreul J., A ordem médica. São Paulo, Brasiliense, 1983.

54. Bench R.J., "Health Science, Natural Science, and Clinical Knowledge". The Journal of Medicine and Philosophy 14:147-164, 1989.

55. En passant, notem aqui a origem baconiana da noção de "história natural das doenças", tão cara à ideologia da medicina preventiva (Arouca 1975).

56. Salmon W.C., "La justificación pragmática de la inducción". In: Swinburne R (org.) La Justificación del Razonamiento Inductivo. Madrid, Alianza Editorial, 1976, p. 105-118. 
Não obstante, o emprego de ramos aplicados da matemática, como o cálculo, a teoria das probabilidades e a estatística, mostrava-se atraente e adequado para a abordagem sistemática, a descrição precisa e o desenvolvimento analítico dos objetos de conhecimento do campo da biologia, com imediatas repercussões na clínica. Um exemplo histórico e familiar é bastante ilustrativo da questão: descendente de uma família de influentes matemáticos, Daniel Bernoulli (1700-1782) foi um físico, matemático e médico suiço que, como nos contam Lilienfeld \& Lilienfeld (1980), influenciou o aparecimento da "aritmética médica". A principal contribuição de tão ilustre precursor terá sido a proposta de emprego da recém-nascida teoria dos jogos de Pascal-Bernoulli (Jacques Bernoulli, seu tio paterno) para o aperfeiçoamento dos métodos diagnósticos e prognósticos da então nascente clínica.

Uma das primeiras etapas na constituição da clínica moderna incorporou uma séria tentativa de quantificar o processo de tomada de decisões, através do cálculo do que veio a se chamar "grau de certeza". A questão da segurança diagnóstica foi, naquela fase, essencial para o estabelecimento da clínica como uma "ideologia científica". Conforme Foucault, ${ }^{58}$ nos primórdios da clínica moderna propunha-se que todo e qualquer diagnóstico ou prognóstico seria uma totalidade divisível em tantas certezas quantas fossem possíveis de ser estabelecidas pela experiência clínica. Trata-se, evidentemente, de uma aplicação bastante ingênua da noção de probabilidades. Porém, é inegável que traz uma confortável concepção cumulativa da tarefa diagnóstica. Cada sinal e sintoma desvendado pelo exame clínico ou pela anamnese acrescentaria mais "graus de certeza" à exploração científica da doença do paciente.

Um interessante exemplo do modo como operava esta primitiva "clínica probabilística”, datado do ano 10 da Revolução Francesa, é assim narrado:

"Um doente que consultara Brulley desejava ser operado de cálculo; a favor da intervenção, duas probabilidades favoráveis: o bom estado da vesícula e o pequeno volume do cálculo; mas, contra elas, quatro probabilidades desfavoráveis: o doente é sexagenário; é do sexo masculino; tem um temperamento bilioso; está afetado por uma doença de pele. $O$ indivíduo não quis entender essa aritmética simples: não sobreviveu à operação". ${ }^{59}$

57. Escher R., Methodological Pragmatism. New York, Nova York Univ. Press, 1977.

58. Foucault M., op. cit, 1979.

59. Foucault M., op. cit, 1979, p. 118. 
O cálculo aditivo de probabilidades clínicas de fato estrutura-se como um método inaugural de análise dos sintomas e sinais, talvez a primeira forma sistemática de análise propedêutica, destacando-se da clínica classificatória da medicina das espécies. A baixa eficácia de tal sistema certamente não foi o principal fator responsável pelo seu paulatino abandono, substituído pela "arte \& prática" da medicina moderna, principalmente porque a clínica daquela época não se vinculava particularmente a uma busca de resultados, e sim privilegiava mais o potencial totalizador das suas práticas e procedimentos. ${ }^{60}$

No nível conceitual, o desenvolvimento de modelos mecânicos causais no contexto da fisiopatologia desloca (e reprime) com facilidade o enfoque mais elástico e flexível de uma clínica baseada em predições com algum grau mensurável de incerteza. Não é por acaso que justamente Claude Bernard, principal teórico da "medicina científica", será o maior crítico do uso de qualquer modalidade de raciocínio probabilístico na ciência médica, advogando o emprego de modelos tipo processo causal, com um forte componente sistêmico. ${ }^{61}$

O diagnóstico, enquanto processo heurístico típico da clínica médica, resulta de um raciocínio de probabilidades somente no sentido de que aí se investiga o que será provável. Porém, isto não significa pensar em termos probabilísticos, pelo menos não no sentido restrito de "cálculo matemático das probabilidades" dentro da teoria dos jogos, enquanto freqüência de ocorrências em relação à totalidade de eventos possíveis. Trata-se, na verdade, de usar o termo probabilidade no sentido de aceitabilidade, plausibilidade ou corroboração de uma hipótese clínica diagnóstica, tal como faz o senso comum.

Popper, ${ }^{62}$ analisando esta importante questão em um outro contexto, propõe a distinção, que pode nos ser útil, entre uma interpretação objetiva e uma interpretação subjetiva da noção de probabilidade. De acordo com ele:

"A interpretação subjetiva é sobretudo o resultado da idéia plausível e largamente difundida de que sempre que a probabilidade intervém nas nossas considerações isso se deve ao nosso conhecimento imperfeito: se o nosso conhecimento fosse perfeito, não precisaríamos da probabilidade, pois disporíamos sempre de certeza. Esta idéia está profundamente errada. (...) Isto acontece porque a teoria subjetiva tenta interpretar $p(a, b)$ (proba-

60. Clavreul J., op. cit.

61. Bernard C., Introduction to the Study of Experimental Medicine (1865). London, Macmillan, 1927.

62. Popper K., A lógica da descoberta científica. Sāo Paulo, Cultrix, 1975. 
bilidade de $a$ dado $b$ ) como o grau de crença em $a$ que pode ser racionalmente justificado pelo nosso conhecimento total e efetivo $b$. Assim, se interpretarmos a interpretação lógica subjetivamente - isto é, em termos do nosso conhecimento ou ignorância - então $p(a, b)$ passará a ser, precisamente, o grau a que o nosso conhecimento total efetivo $b$ justifica racionalmente um $a$ dúbio ou hipotético."63

Nesta perspectiva, a probabilidade objetiva define-se em termos de freqüência e tendência de eventos em uma seqüência virtual computável, enquanto a probabilidade subjetiva é interpretada no sentido de dependente do estado de conhecimento. Para o que nos interessa, trata-se de admitir que qualquer uso subjetivo do conceito de probabilidade, tanto no sentido causalista etiológico quanto para o reconhecimento da correspondência sinal/sintoma e diagnóstico, não poderá ser legitimado pela mesma lógica fundamental do cálculo matemático das probabilidades objetivas. Em outras palavras, a lógica oriunda da interpretação objetiva da teoria probabilística não será capaz de sustentar a abordagem clinimétrica. Portanto, a expressão do grau de certeza da clínica segundo os termos da probabilidade objetiva é estranha ao raciocínio clínico, estruturado sobre modelos causais sistêmicos ou mecânicos.

Isto ocorre porque, no âmbito da teoria das probabilidades, a aleatoriedade e a independência são axiomas fundamentais. Define-se um evento como probabilístico quando, por sua independência em relação a ocorrências prévias ou equivalentes, e dada a sua regência pelo acaso (o que implica também ausência de desvio, direção ou tendenciosidade), as probabilidades das suas conseqüências são recuperadas integralmente a cada nova ocorrência. Então, se eu lanço uma moeda, as probabilidades de cara e coroa serão igualmente 0,50 ; a cada nova jogada, estas probabilidades serão restauradas. Caso consideremos seqüências definidas de jogadas, as probabilidades de repetição serão dependentes dos eventos anteriores, porém tal dependência resulta da decisão de considerar um único lançamento, dois ou uma série finita de lançamentos. Mesmo assim, as probabilidades das jogadas individuais são rigorosamente as mesmas, torno a repetir, restauradas a cada novo arremesso.

Quando se produz uma série de observações em uma população (o que se constitui na prática típica da pesquisa epidemiológica), as relações entre o evento observado e o processo ou fenômeno que se supõe seu fator são primariamente interpretadas como independentes e aleatórias, regidas exclusivamente pelo acaso. Ou seja, testa-se em primeiro lugar os pressu-

63. Popper K., op. cit., p. 300. 
postos de um modelo estocástico abstrato, construído a partir de relações puras, e os nexos encontrados, caso tais supostos sejam satisfeitos pela configuração dos dados produzidos. Quando o modelo de base observacional se revela desviante em relação ao modelo estocástico original, propõe-se uma estrutura de explicações de natureza determinística, capaz de melhor ajustar a série empírica observada.

\section{Comentário final (sobre pretensões)}

A prática concreta dos sujeitos sociais precede a sua produção discursiva, prioritariamente feita de expectativas e desejos investidos sobre objetos idealizados de conhecimento e intervenção. Neste caso, mesmo os discursos mais eloquentes, atrativos e aparentemente razoáveis mostram-se impotentes perante a lógica interna dos campos científicos e a prática efetiva dos seus intelectuais.

Clínicos e epidemiologistas parecem pretender certas realizações que se encontram além do alcance do seu instrumental lógico-racional. Se, por um lado, alegam fazer coisas que, de fato, nunca poderiam fazer, por outro lado têm alcançado o que não parecia ser possível. De todo modo, observo a facilidade (ou leviandade) com que os epidemiologistas asseguram que pensam em termos de causalidade e que produzem um saber sobre causas. Os manuais epidemiológicos falam até mesmo de critérios de atribuição de causalidade. Mas, como vimos, na verdade a prática heurística da epidemiologia enquanto disciplina científica simplesmente não autoriza a construção de enunciados com tal grau de positividade, ${ }^{64,65}$ na medida em que se ancora em um modo de raciocínio essencialmente probabilístico. No caso específico, por mais que os clínicos valorizem a proposta de uma clinimetria capaz de projetar uma probabilidade objetiva sobre o construto diagnóstico-prognóstico, ${ }^{66}$ não conseguirão produzir nem operar um saber probabilístico autêntico. Sua prática concreta não possibilita a operação de modelos probabilísticos. Sua prática concreta não possibilita a operação de modelos probabilísticos.

No que se refere à luta pela "hegemonia teórica" no campo disciplinar da saúde, acredito que a epidemiologia não é o único (nem mesmo o principal) alvo da epidemiologia clínica. A própria clínica médica, enquanto prática científica peculiar por sua capacidade integrativa e totali-

64. Wallace W., "The Decline and Fall of Causality". In: Causality and Scientific Explanation. Ann Arbor, University of Michigan Press, 1974, p. 165-97.

65. Weed D., op. cit.

66. Jénicek M. e Cléroux R., op. cit. 
zadora, não poderá sobreviver enquanto tal a uma ofensiva tecnificadora de tal ordem. No momento, a epidemiologia clínica parece investir em uma certa aliança tática com a clínica contra a epidemiologia. Caso a ofensiva da epidemiologia clínica seja relativamente bem-sucedida, a epidemiologia poderá recuar para a sua posição convencional de "ciência básica da saúde pública”. Nesta hipótese, não nos deixemos enganar, o resultado será a destruição da clínica tal como a conhecemos.

\section{RESUMO}

\section{Prática Clínica, Epidemiologia e Epidemiologia Clínica}

O presente estudo discute as múltiplas articulações conceituais e metodológicas entre a prática clínica e a epidemiologia, afirmando que o modo de produção do saber epidemiológico é inadequado para a formação do discurso clínico. Posteriormente ele avalia o grau de legitimidade epistemológica da proposta de hibridação consolidada na chamada "epidemiologia clínica", especialmente no que diz respeito à tentativa de tornar técnica a decisão corrente em abos os campos do diagnóstico e da terapêutica da prática clínica, que ficou conhecida como "clinimetria".

\section{ABSTRACT}

\section{Clinical Practice, Epidemiology and Clinical Epidemiology}

This study debates the multiple conceptual and methodological linkages between clinical practice and epidemiology, alleging that the mode of production of epidemiological knowledge is inadequate for the making of the clinical discourse. It subsequently assesses the degree of epistemiological legitimacy of the proposal of hybridization which consolidates into the so called "clinical epidemiology", specially in relation to its attempt to technify the decision making process in both the diagnostic and therapeutic fields of the clinical practice, which came to be known as "clinimetry". 


\section{RESUME}

\section{Pratique clinique, epidemiology et épidémiologie clinique}

Ce texte discute les rapports conceptuels et méthodologiques entre la pratique clinique et l'épidémiologie. Cette discussion mène l'auteur à la conclusion suivante: le mode de production de la connaissance épidémiologique n'est pas convenable pour la formation du discours clinique.

Ce texte s'occupe aussi d'une analyse épistémologique de l'épidémiologie clinique et de la pratique clinique. 
. 